\title{
Situación alimentaria de pacientes pediátricos hospitalizados
}

\author{
V. Gattás, ${ }^{1,3}$ A. Fuentes, ${ }^{2,4}$ S. Japaa. ${ }^{2.4}$ y R. Uauy 1.4 .5
}

\author{
ABSTRACT \\ Diets of pediatric hospitalized patients in a General Metropolitan Hospital.
}

\begin{abstract}
To evaluate the adequacy of food intake for inpatients of a Pedjatric Hospital, the actual ingestion of 161 patients was shudied using a 3 day record of intake. Their ages raviged from 2 months to 16 years. The amount of protein, carbohydrate, lipids and calories ingested daily was talcuted tor each patient based on the composition of the standard hospital diets. These values were compared with the USA NAS NRC RDA/74 adfusted for weight and age given for healthy children. The results obtained, showed that $87.5 \%$ of the propulation surveyed were receiving inaderyuate diets even for norinal children. Mean \pm S.D. intake for proteis was $116 \pm 54 \%$ and for eneng was $61.5 \pm 25 \%$ of the standard recommendations. Close to $90 \%$ of all patients received insufficient energy and $50 \%$ low protein intakes. Calories derived from proteins $(\mathbf{P} \%)$ acconted for $16 \%$ ant those denived from lipids $(\mathbf{F} \%)$ for $14 \%$ of total energy intake, giving a $\mathrm{F} \% / \mathrm{P} \%$ ratio of $\mathrm{t} .6$ which is lower than the recommended value of 2.5 . A significant weight loss was encountered in patients receiving less than $96 \%$ of the ADA's for prolein and calories. These results show that hospital diets do not cover the nutritional requirements of healthy chiddren and less so the needs of the ill. It is imperative to monitor nutritional status of hospibalized patients and to provide an adequate nutritional support to avoid malnutrition related morbidity and mortality.
\end{abstract}

La dieta en el paciente pediátrico hospitalizado debe cumplir un rol importante como es evitar el deterioro del estado nutricional y los cambios adversos en composición corporal que la desnutrición acarrea: El aporte dietario debe proveer al enfermo de los nutrientes esenciales y no esenciales necesarios para una recuperación óptima. EI objetivo final de su adecuación es la mantención del compartimento magro visceral y muscular para lograr una mejor capacidad defensiva frente a la infección y una mayor capacidad regeneradora de tejidos a fin de disminuir la estadía y posibilitar la recuperación rápida del enfermo. ${ }^{1}$

Las recomendaciones alimentarias de la infancia se fundamentan en estudios efectuados en ninos normales y sanos. ${ }^{23,4}$ Estas mismas recomendaciones energéticas, proteicas y lipidicas, se utilizan habitualmente para los pacientes hospitalizados, asumiéndose a priori un ajuste equivalente al ahorro energético por situación de reposo que se supone cubriria el mayor requerimiento calórico de la patología subyacente. El rol que juega la infección en la nutrición del individuo también ha sido estudiado a nivel poblacional, donde se ha encontrado correlaciones negativas entre ingesta energética de patologia infecciosa en menores de

\footnotetext{
${ }^{1}$ Centro de Investigación Clínica INTA. Universidad de Chile.

2 Hospital Luis Calvo Mackenna

${ }^{3}$ Nutricionista

${ }^{4}$ Medico Cinjuno

5 Doctorado en Bioquímica Nutricional.
}

tres años. ${ }^{5}$ Además se ha demostrado que la infección es una causa frecuente de mala absorción, utilización disminuida de nutrientes y de pérdida de peso..$^{6.7}$ este ciclo de infección, anorexia, depleción corporal, mayor susceptibilidad de infección, debe romperse al tratar la desnutrición y sus consecuencias. Otro factor que influye en la ingesta alimentaria es la anorexia que acompaña patologias específicas, ${ }^{8}$ lo que implica disminuir el voIumen de lo ingerido $y$ por ende una menor cobertura de los requerimientos, como consecuencia de esto la dieta del enfermo debería poseer una densidad de nutrientes variable adecuada a cada tipo de patología. Por otra parte no debe olvidarse que el niño es un organismo en crecimiento $y$ por tal concepto debe estar cubierto con un aporte nutricional que permiten un crecimiento pondo-estatural correspondiente a la edad.

La presente investigación tuvo por objeto conocer el aporte nutritivo de alimentos y preparaciones que componen la dieta hospitalaria. Determinar la ingesta real de alimentos y preparaciones por parte del paciente y evaluar la adecuación de la ingesta con las recomendaciones individuales. Entregar los antecedentes necesarios gue permitan implementar programas para mejorar la situación nutricional del enfermo hospitalizado.

\section{MATERIAL Y METODO}

Para cumplir con los objetivos del presente estu- 
dio se realizó una encuesta alimentaria de tres dias consecutivos, que abarcó a un total de 158 pacientes, cuyas edades fluctuaban entre 2 meses y 16 años, en un hospital pediátrico del Area Metropolitana. Un $73 \%$ de la muestra correspondió a pacientes del servicio de Medicina y un $33 \%$ de Cirugía.

Para determinar la alimentación de estos pacientes se realizó un estudio directo de las preparaciones y dietas entregadas por el Servicio de Alimentación de dicho hospital. La información de minutas se recogió directamente de los profesionales que tienen a su cargo la alimentación, $y$ por otra parte se corroboró mediante un análisis químico de las dietas tomadas al azar durante los tres días que duró la experiencia. La ingesta de los pacientes se obtuvo a través de un formulario diseñado de acuerdo con el objetivo de la investiga- ción y que consideraba la ingesta real de alimentos de 3 días consecutivos. En los lactantes se consignó el volumen real de alimentación líquida ingerida; en los preescolares y escolares volumen y peso de preparaciones líquidas y sólidas, respectivamente, recibidas. El aporte nutritivo de calorías, proteínas, grasa e hidratos de carbono para cada niño se calculó en base a tablas a partir de los valores obtenidos de volumen y peso ingerido. Los valores de ingesta real se compararon con las recomendaciones del NRC NAS 1974 EE.UU. para individuos sanos. ${ }^{10}$ Realizándose un ajuste por patologías según importancia del estado catabólico y pérdidas nutricionales en cada patología, aplicando los factores de corrección para Calorías y Proteínas que se observan en la Tabla 1."

Tabla 1

Factores de correcoión para energia y proteina, evaluación del estado nutricional H.L.C.M., 1978

\begin{tabular}{|c|c|c|c|c|c|}
\hline & & CATEGORIA & $N$ & ENERGLA & PAOTEINA \\
\hline & I & Febriles - de 7 días & 65 & $\times 1.5$ & $\mathrm{x} 1.7$ \\
\hline \multirow[t]{2}{*}{ Médicos } & Il & Febriles + de 7 días & 8 & $\mathrm{x} 0.8$ & $\times 1.3$ \\
\hline & I1I & Afebriles & 42 & $\times 0.8$ & $\times 1.3$ \\
\hline \multirow[t]{4}{*}{ Quinírgicos } & IV & Agudos abdominales & 12 & $\times 1.5$ & $\times 1.7$ \\
\hline & $V$ & C.V. Urológicos digestivos & 9 & $x 1.3$ & $\times 1.5$ \\
\hline & VI & Ortopédicos y traumatol. & 16 & $\times 0.8$ & $\times 1.3$ \\
\hline & YII & Cinugía plâstica & 6 & $\times 1.0$ & $\times 1.3$ \\
\hline
\end{tabular}

Tabla 2

Características del estado nutricional de pacientes según grupos de patologías

\begin{tabular}{|c|c|c|c|c|c|c|c|c|c|c|c|c|c|}
\hline \multirow{2}{*}{$\begin{array}{l}\text { PATOLOGIAS } \\
\text { Médicas }\end{array}$} & \multirow[t]{2}{*}{$N$} & \multicolumn{2}{|l|}{$\begin{array}{l}\text { EDAD } \\
\text { Años }\end{array}$} & \multicolumn{4}{|c|}{ PESO/EDAD } & \multicolumn{6}{|c|}{$\begin{array}{c}\text { ESTADO NUTHICIONAL } \\
\% \text { DEL STANDARD* } \\
\text { PESO/TALLA TALLA/EDAD }\end{array}$} \\
\hline & & $\overline{\mathbf{x}}$ & & D.E. & $\overline{\mathbf{x}}$ & & D.E. & $\overline{\mathbf{x}}$ & & D.E. & $\overline{\mathbf{x}}$ & & D.E. \\
\hline $\begin{array}{l}\text { Febril }<7 \text { dias } \\
\text { Febril }>7 \text { días }\end{array}$ & $\begin{array}{r}65 \\
8\end{array}$ & $\begin{array}{l}0.9 \\
5.1\end{array}$ & \pm & $\begin{array}{l}0.6^{\circ} \\
2.9\end{array}$ & $\begin{array}{l}80 \\
84\end{array}$ & $\begin{array}{l} \pm \\
\pm\end{array}$ & $\begin{array}{l}17 \\
13\end{array}$ & $\begin{array}{l}91 \\
88\end{array}$ & $\begin{array}{l} \pm \\
\pm\end{array}$ & $\begin{array}{l}18 \\
16\end{array}$ & $\begin{array}{l}93 \\
96\end{array}$ & $\begin{array}{l} \pm \\
\pm\end{array}$ & $\begin{array}{l}6 \\
5\end{array}$ \\
\hline $\begin{array}{l}\text { Afebril } \\
\text { Quirúrgicos }\end{array}$ & 42 & 4.2 & \pm & 2.3 & 74 & \pm & 17 & 87 & \pm & 13 & 92 & \pm & 7 \\
\hline $\begin{array}{l}\text { Agudos, abdoninales } \\
\text { Cardiovasculares, }\end{array}$ & 12 & 6.9 & \pm & 3.1 & 88 & \pm & 18 & 100 & \pm & 19 & 95 & \pm & 6 \\
\hline urológicos, digestivos & 9 & 6.3 & \pm & 4.9 & Bo) & \pm & 13 & 97 & \pm & 14 & 92 & \pm & 8 \\
\hline Fracturas, ortopedicas & 16 & 6.9 & \pm & 3.0 & 78 & \pm & 31 & 76 & \pm & $19 b$ & $\mathbf{9 5}$ & 3 & 6 \\
\hline Cirugía plústica & 6 & 2.1 & \pm & 1.8 & \multicolumn{3}{|c|}{ SIV DATOS } & \multicolumn{3}{|c|}{ SIN DATOS } & \multicolumn{3}{|c|}{ SIN DATOS } \\
\hline
\end{tabular}

ap< 0.01 diferencia de edad con respecto a los 5 grupos restantes.

${ }^{b} \mathrm{p}<0.01$ diferencia de P/T con respecto a los 5 grupos restantes.

* Standard: Mediuna de indicador antropométrico en tablas del Centro Nacional de Estadisticas de Salud, Estados Unidos, 1976. (NCHS-USA. 76). 
El análisis químico consideró la dieta de un día para lactantes, preescolares y escolares, analizándose calorías totales, proteínas, grasa, hidratos de carbono y cenizas. La energía fiue deteminada por Bomba calorimétrica macro Kjeldahl para proteínas, método de Soxlet para grasa, los hidratos de carbono se calcularon por diferencia y cenizas por calcinación en mufla a $105^{\circ}$ de acuerdo a metodología habitual del INTA previamente detallada. ${ }^{12}$ Paralelamente a la evaluación alimentaria se efectuó un estudio de la situación nutricional de la misma población que incluyó indices antropométricas como, peso, talla, cireunferencia braquial y pliegue tricipital ${ }^{13} \mathrm{e}$ indicadores de laboratorio como hematocrito por micrométodo, albumina ${ }^{14}$ y pruebas cutáneas.

\section{RESULTADOS}

Al analizar las fórmulas alimentarias utilizadas en el hospital se observa una diferencia en contenido energético a favor de la fórmula $\mathrm{L} 2$ con respecto a la formula $L l$, lo que se puede observar claramente en la tabla N. ${ }^{\circ}$. En esta tabla se entregan los valores de aporte porcentual de proteinas y grasas сол respecto a calorias de las dietas correspondientes a un periodo de 8 dias. El P\% de estas minutas es de $14.8 \pm 0.2$ con un $G \%$ de $20.8 \pm 0.7$ lo que da una relación de 1.4 de calorías de origen graso con respecto a calorias de origen proteico.

Al analizar la adecuación de la ingesta por patologías podemos observar en la tabla 4 que las Calorías cubren en promedio un $61.6 \%$ de lo reco-

Tabla 3

Densidad $y$ tipo de energia de dietas utilizadas en el hospital

\begin{tabular}{lcccccc}
\hline & \multicolumn{2}{c}{$\begin{array}{c}\text { APORTE ENERGETICO } \\
\text { Kcal/ml }\end{array}$} & \multicolumn{4}{c}{ PORCENTAJE DE ENERGIA DEFIVADA } \\
& Kcal/dia & GLUCIDOS PROTEINAS & GRASAS & G/P \\
\hline Fórmula láctea I & 70 & & 64.5 & 12 & 23.5 & 1.96 \\
Fómula láctea II & 88 & & 52.8 & 14.5 & 32.7 & 2.25 \\
$\begin{array}{l}\text { Dieta Mixta liquida } \\
\text { Dietas para }\end{array}$ & 82 & & 45.3 & 7.3 & 47.4 & 6.5 \\
Preescolares & & $2.116 \pm 37$ & 64.5 & 14.8 & 20.7 & 1.4 \\
\hline
\end{tabular}

GLUCIDOS: Recomendación por Fomon 29-63\%

PROTEINAS: Recomendación por Fotuon 7-16\%

GRASAS: Recomendación por Fomon $30-55 \%$

Tabla 4

Distribución de adecuación enengética y protéica según gnupos de patologłas

\begin{tabular}{|c|c|c|c|c|c|c|c|c|c|c|}
\hline \multirow{4}{*}{ PATOLOGLAS } & \multirow{4}{*}{$\mathbf{N}$} & & & & \multicolumn{6}{|c|}{ ADECUACION } \\
\hline & & \multirow{2}{*}{\multicolumn{3}{|c|}{$\begin{array}{c}\text { EDAD } \\
\text { Años }\end{array}$}} & \multicolumn{3}{|c|}{ ENERGIA } & \multicolumn{3}{|c|}{ PAOTEINA } \\
\hline & & & & & \multicolumn{6}{|c|}{ \% STANDARD* } \\
\hline & & $x$ & & D.E. & $x$ & & D.E. & $x$ & & D.E. \\
\hline \multicolumn{11}{|l|}{ Médicas } \\
\hline Febriles $<7$ dias & 65 & 0.9 & \pm & $\mathbf{l}^{\mathbf{b}}$ & 54 & \pm & $13 a$ & 89 & \pm & $52^{3}$ \\
\hline Febriles $>7$ dias & 8 & 5.1 & \pm & 3 & 64 & \pm & 27 & 119 & \pm & 50 \\
\hline Afebriles & 42 & 4.2 & \pm & 2 & 67 & \pm & $21^{2}$ & 134 & \pm & 453 \\
\hline Médico total & 115 & & & & 59 & \pm & 24 & 107 & \pm & 54 \\
\hline \multicolumn{11}{|l|}{ Quinúrgicos } \\
\hline Agudos y abdominales & 12 & 6.9 & \pm & 3 & 58 & \pm & 30 & 121 & \pm & 39 \\
\hline Cardiovasculares, & & & & & & & & & & \\
\hline urologicos y digestivos & 9 & 6.3 & \pm & 5 & 66 & \pm & 23 & 129 & \pm & 61 \\
\hline Fracturas, ortopedicos & 16 & 6.9 & \pm & 3 & 76 & \pm & 29 & 141 & \pm & 52 \\
\hline Cirugía plâstica & 6 & 2.1 & \pm & $2 b$ & 69 & \pm & 29 & 151 & \pm & 47 \\
\hline Quirúrgico total & 43 & & & & 68 & \pm & 29 & 138 & \pm & 50 \\
\hline
\end{tabular}

${ }_{\mathrm{a}}^{\mathrm{a}}<0.001$ diferencia de adecuación entre pacientes febriles 7 días $y$ afebriles.

${ }^{b} p<0.01$ diferencia de edad con los 5 grupos restantes.

* Recomendaciones NAS-NRC 1974 (10). 
mendado según peso-edad para individuos sanos, mientras que la proteina cubre en promedio $115.7 \%$ de lo recomendado para estos mismos grupos. El grupo más desfavorecido corresponde a los febriles de menos de 7 dias, obteniéndose una diferencia significativa ( $\mathrm{p}<0.000 \mathrm{~L}$ ) entre este grupo y el grupo de pacientes afebriles; para el caso de proteínas se observa una diferencia significativa $(\mathrm{p}<0.001)$ entre pacientes médicos y quirúrgicos a favor de los pacientes quirúrgicos $(138.2<50.3)$.

Para los efectos de corrección de Calorias y Proteínas segin patología se aplicaron los factores que se indican en la tabla I. La adecuación de proteina y energia corregida se observa en la tabla 5 , indicando para energía que el $85 \%$ de los pacientes médicos y el $67 \%$ de los quirúrgicos cubren menos del $50 \%$ de las recomendaciones estableciéndose diferencias significativas entre pacientes médicos y quinúrgicos $(\mathrm{p}<0.01)$. En lo que respecta a proteinas, se observa que los pacientes médicos cubren sólo un $74 \%$ de lo recomendado difiriendo significativamente de los quirírgicos que lo hacen en un $94 \%$ p $<0.01$ ).

La Figura 1 nos muestra la distribución de los pacientes médicos y quinírgicos en relación a la

\section{Tabla 5}

Distribución de adecuación energética y proteica corregidas por tipo de patologías

PATOLLGIAS

Médicas

Febriles $<7$ días

Febriles $>7$ dias

Afebriles

Total

Quinirgicas

Agusłos abdominales

C.V. Utológicos,

digestivos

Fracturas, ortopedico

Cirugia plástica

Total
$\mathrm{N}$

\begin{tabular}{|c|c|c|c|c|c|c|}
\hline & $\overline{\mathrm{x}}$ & & D.E. & $\overline{\mathbf{X}}$ & & D.E \\
\hline 65 & .34 & \pm & $\mathbf{L} 5^{h}$ & 53 & \pm & $31^{b}$ \\
\hline 8 & 44 & \pm & 22 & 91 & \pm & 38 \\
\hline 42 & 45 & \pm & 16 & 103 & \pm & 35 \\
\hline 115 & 39 & \pm & $16^{4}$ & 74 & \pm & $41^{\prime \prime}$ \\
\hline 12 & 44 & \pm & 23 & 71 & \pm & 23 \\
\hline 9 & 47 & \pm & 19 & 86 & \pm & 41 \\
\hline 16 & 52 & \pm & $2 L^{b}$ & 115 & \pm & $40^{b}$ \\
\hline 6 & 48 & \pm & 22 & 89 & \pm & 28 \\
\hline 43 & 48 & \pm & $21^{\circ}$ & 94 & \pm & $38^{\mathrm{a}}$ \\
\hline
\end{tabular}

a = Diferencia significativa p 0,01 entre pacientes médicos y quinírgicos.

$\mathrm{b}=$ Crupos extremos donde la diferetleia significativa es mayor aproximándose a un $\mathrm{p}<0.001$.

\section{Figura 1}

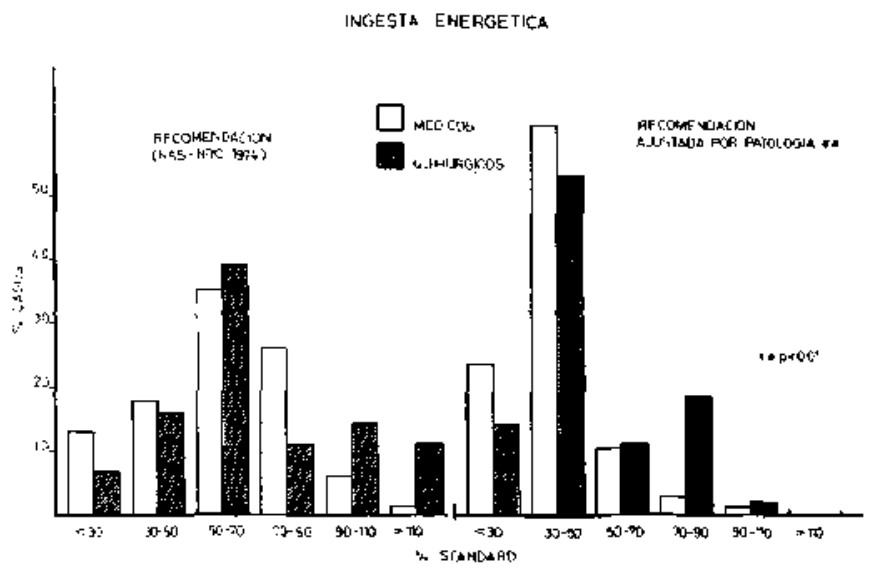

Figura 1. Distribución de paciertes médicos y cuinirgicos según adtecuación de recomendacich energética $\{a$ ) y corregido por patologia (b). Eje vertical porcentaje de casos, Eje horizontal \% de estándard recomendado.

${ }^{* *}=\mathbf{p}<0,0$ l diferencia significativa entre Recomendación energética estándard y conregida. 
adecuación de la ingesta energética con y sin ajuste seguin patologia. No se observan diferencias importantes entre pacientes médicos y quirúrgicos; pero sí es interesante destacar que existe un $11 \%$ de pacientes que estín por debajo del $30 \%$ de las recomendaciones standard, un $13 \%$ de pacientes que cubren sus recomendaciones; y de estos últimos un $4 \%$ que está por sobre el standard. A] corregirse por patología este último grupo desaparece y disminuye el grupo nomal, aumentando el grupo que cubre sus necesidades entre un 30 y $50 \%$ en relación al standard. EI 85\% de los pacientes médicos y el $67 \%$ de los quirúrgicos cubren menos del $50 \%$ de las recomendaciones energéticas corregidas.

La Figura 2 nos da la distribución de la ingesta proteica de los pacientes médicos y quinírgicos en relación al standard de recomendación y luego de ajustado por patologías. En promedio los pa- cientes médicos están cubiertos en un $107 \% \pm 54$ en relacion al standard. $\mathbf{A}$ los extremos se destaca un $10 \%$ de pacientes bajo el $40 \%$ de lo recomendado lo que signífica estar recibiendo menos de $1 \mathrm{~g}$ de proteína por $\mathrm{kg}$ de peso; y un $38 \%$ de pacientes que está por solve el $120 \%$ del standard. Los pacientes quirúngicos cubren en un $138 \%$ de sus necesidades, existiendo gnupos que estan fuera de las recomendaciones. Destacándose la presencia de un $24 \%$ de pacientes que están por sobre el $160 \%$ de las recomendaciones y un $25 \%$ que estí bajo el $40 \%$ de lo recomendado. Estos grupos médicos y quirúrgicos difieren significativamente ( $\mathrm{p}$ $<0.001$ ). Al corregir las recomendaciones por los factores descritos en la tabla I los pacientes médicos cubren en promedio sólo un $73.5 \%$ de las recomendaciones para proteínas, difiriendo significativamente de los quinúrgicos que lo hacetı en un $94 \%(p<0.01)$.

Figura 2

INGESTA PROJEICA

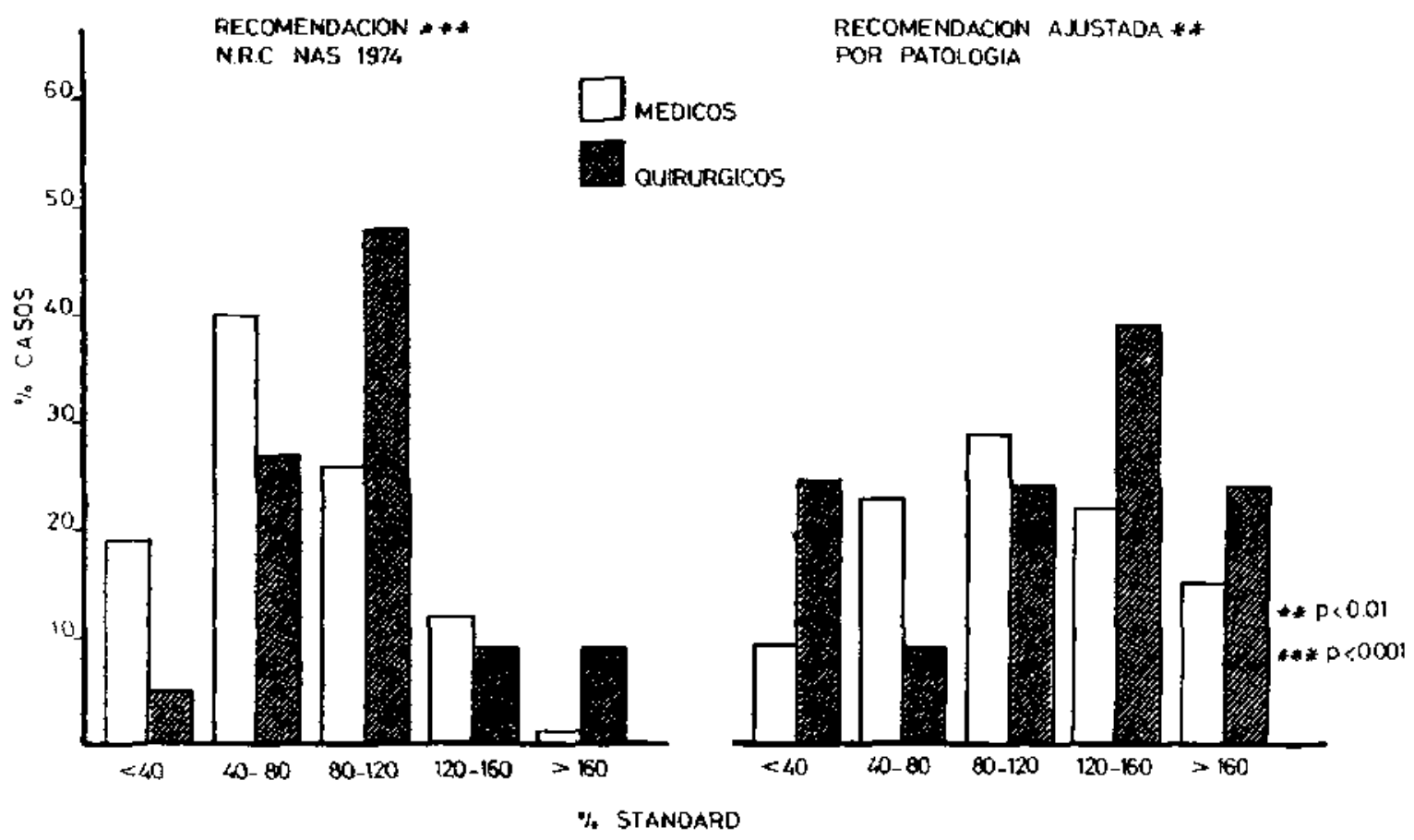

Figara 2. Distribución de pacientes médicos y quirúrgicos, segün adecuación de recumendación proteica (a) y corregidal por patologia (b). Eje vertical porcentaje de casos y Eje horizontal porcentaje del estíndard.

** $p<0.01$ Difereacia significubiva entse parientes médicas y quirürgicos segün recamendación proteica.

-** $\mathrm{p}<0,001$ Diferencia signifimava entre pacientes médicos y quirírgicos, según recomendación proteica corregida por patolugia. 
La Tabla 6 describe la evolución del estado nutritivo según condición al ingreso de 133 pacientes médicos y quínírgicos con peso de nacimiento mayor de 2.500. De 62 pacientes que in-

\section{Tabla 6}

Evolución del estado nutritivo según condición atl ingreso en pacientes médicos y quinirgicos Hospital Luis Calvo Mackenna, 1978

\begin{tabular}{lccc}
\hline $\begin{array}{c}\text { ESTADO NUTRITIVO } \\
\text { INGRESO }\end{array}$ & \multicolumn{2}{c}{$\begin{array}{c}\text { ESTADO NUTRITIVO } \\
\text { AL ESTUDIO }\end{array}$} \\
\hline & Nonnal & Desnutrido & Total \\
Nontial & 41 & 21 & 62 \\
Desnutrido & 4 & 67 & 71 \\
\hline \multicolumn{1}{c}{ TOTAL } & 45 & 88 & 133 \\
$\mathrm{x}^{2} 54.1$ & & & \\
$\mathrm{p}<0.001$ & & &
\end{tabular}

Normal $P / E>90 \%$ del standard

Desnutrido $\mathrm{P}, \mathbf{E}<90 \%$ del standard gresan normales (peso-edad $<90 \%$ ) 21 caen a desnutridos en el momento de] estudio, y de 71 desnutridos al ingreso (peso-edad $<90 \%$ ) al momento del estudio sólo hay 4 recuperados $\left(x^{2}=\right.$ 54.1 p $<0.001$ ). La tabla 7 nos muestra la relación entre variación ponderal y grado de adecuación de la ingesta a las recomendaciones para energía en 106 de 133 pacientes detallado en el párrafo anterior. Vemos que de 11 pacientes que reciben más del $90 \%$ de las recomendaciones de energía 8 han ganado peso en el momento del estudio y que para 95 que reciben menos del $90 \%, 71$ estan perdiendo peso $\left(x^{2} 10.5 \mathrm{p}<0.01\right)$.

La tabla 8 muestra la relación entre variación ponderal $y$ adecuación de la ingesta a las recomendaciones para proteinas en 104 pacientes médico-quinírgico. En los pacientes que reciben menos de $90 \%$ de lo recomendado 35 de 40 (88\%) pienden peso, contra 37 de $64(57 \%)$ que reciben más del $100 \%$ de las recomendaciones para proteí$\operatorname{nas}\left(x^{2} 10.2 p<0.01\right)$.

Tabla 7

Relación entre variación ponderal y adecuación de la ingesta de energía. H.L.C.M., 1978

VARIACION PONDERAL

INGESTA DE ENERGIA

DE \% DE LO RECOMENDADO*

gaNan PESO PIEADEN PESO

TOTAL

\begin{tabular}{rrrrr}
$<90$ & 24 & 71 & 95 \\
$\geq 90$ & 8 & 3 & 11 \\
\hline TOTAL & 32 & 74 & 106
\end{tabular}

$x^{2} 10.5$

$\mathrm{p}<0.01$

* Pc50 para recomendación de energía según edad.

R.D.A. (NAS-NRC-U.S.A.) 1974.

Tabda 8

Relación entre variación ponderal y adecuación de la ingesta de proteina H.L.C..M., 1978

\begin{tabular}{cccc}
\hline $\begin{array}{l}\text { INGESTA DE PROTEINA } \\
\% \text { DE LO RECOMENDADO* }\end{array}$ & \multicolumn{2}{c}{ VARIACION PONDERAL } \\
\hline$<90$ & GANAN PESO & PIERDEN PESO & TOTAL \\
\hline 90 & 5 & 35 & 40 \\
64 & 37 & 72 & 104 \\
\hline TOTAL & 32 & 72 & 37 \\
\hline
\end{tabular}

\footnotetext{
$\mathrm{x}^{2} 10.2$

$p<0.01$

*Pc50 para recomendación de proteína según edad.

R.D.A. (N.A.S. - N.R.C. U.S.A.) 1974
} 


\section{DISCUSION}

Al analizar los resultados cabe destacar que la densidad y distribución calórica comparada con las recomendaciones propuestas por Fomon, ${ }^{9}$ no se cumple. Se observa una mayor proporción de Calorías derivadas de Hidratos de cartono que calorías grasas, haciéndose más evidente en la fórmula 1. Tanto las fórnulas como las dietas sólidas cubririan las necesidades proteicas, salvo que para la dieta mixta este aporte está cercano al mínimo recomendado, lo que da la posibilidad de caer en déficit si no se ingiere el volumen o cantidad suficiente. Por otra parte para las dietas de preescolares y escolares el aporte calórico diario cubriría en forma apropiada el grupo de niños de hasta 7 a 10 años, siempre que el peso no exceda de $27 \mathrm{~kg}$ y sería inadecuado para edades mayores. ${ }^{\text {10 }}$ También se observa que existe una distribución de la energía ingerida, con un aporte calorico predominantemente derivado de hidratos de carbono (64\%).

Al determinar la ingesta real del paciente nos encontramos que el grupo febril de menos de 7 días compuesto predominantemente por cuadros respiratorios ingiere la menor cantidad de Calorías y proteínas correspondiendo a un $\mathbf{5 4 \%}$ de las recomendaciones standard. Este es el grupo menos cubierto, ya sea por la baja ingesta producto de la enfermedad misma o por el hecho de una distribución inadecuada de los tiempos de alimentación.

En los grupos que predominan preescolares y escolares las recomendaciores proteicas se cubren mejor, no así las calóricas, existiendo, por to tanto, una relación calórica-proteica que no permite la utilización más efectiva de la dieta. El aumentar la grasa en estas dietas mejoraría la ingesta energética sin modificar mayormente el volumen de la dieta. Pretender mejorar la ingesta energética a través de hidratos de carbono necesariamente tropezará con la limitada capacidad gástrica del niño o con la palatibilidad de la ración. Nuestros resultados destacan también la asociación existente entre pérdida de peso $y$ baja ingesta energética lo que refuerza el importante efecto de la densidad y distribución calórica de la fórmula y dietas, respectivamente.

La aplicación de los factores de corrección tuvo por objeto detectar el grupo de pacientes que cubríía mejor sus recomendaciones y con ello ver la utilidad práctica de aplicar estos factores de co- rrección en estudios futuros de apoyo nutricional; su aplicación en este caso nos permitió ver que las recomendaciones de Calorias no se cumplen para ninguno de los grupos. Disminuyendo significativamente la adecuación de las ingestas proteicas, quedando sólo tres grupos cubiertos, tebri] más de 7 días, afebriles y pacientes fracturados y ortopédicos.

La situación alimentaria nutricional descrita y analizada en este trabajo, esta condicionada ya sea por factores derivados del efecto de la enfermedad sobre el paciente o por las características de ìa dieta. ${ }^{18}$ Dentro de los efectos de la enfermedad cabe destacar la anorexia y las alteraciones del gusto y del olfato que determinan ingesta alterada. Por otro lado las diversas patologías pueden modificar los requerimientos y utilización de nu. trientes. En cuanto a la dieta es necesario considerar la calidad, cantidad y aceptabilidad de ellas. Los datos presentados muestran que estos aspectos pudieran ser mejorados sin modificar sustancialmente los costos ya que existe un desequilibrio en el aporte de proteínas en relación a energía. Durante la encuesta de consumo se pudo consignar que otro factor limitante es la falta de personal de apoyo que asegure la ingesta adecuada de la dieta entregada.

\section{CONCLUSIONES}

1. El aporte nutritivo de las dietas de un hospital pediátrico muestra una cantidad, densidad y distribución calórica inadecuada con respecto a recomendaciones estándares.

2. La ingesta real del paciente muestra un aporte energético insuficiente. Solamente se cubre requerimientos de proteínas de una parte de la población estudiada. Existió una relación entre pérdida de peso y un menor aporte energético.

3. Se estima necesario aplicar criterios de corrección por patolngías para dar recomendaciones con un margen de seguridad, ya que las recomendaciones estándares están referidas a sujetos sanos.

4. Existe la necesidad de proporcionar un apoyo nutricional individualizado y de monitorizar los efectos de las dietas sobre el estado nutricional para asegurar una recuperación óptima de la enfermedad.

\section{REFERENCIAS}

I Blackbum, G. L.: Bistrian, B. R.i Mani, B. S.; Schfamm, $y$ 
Smith, M. Nutritional and Metabolic Assesment of the hospitalized patients, J. Purenteral Enteral Nutr. 1: 11, 1977.

${ }^{2}$ Eppright, E. S. and fioderuck, C. 1965. Uiet and nutritional status of lowa school children. Am. J. Public Heatth, 45: 464 471.

${ }^{3}$ Wait, B.; Blair, and Roberts, L. J. 1969. Energy intake of well-nourished childnen and adolexents. An. J. Clin. Nutr. 22: $1333-1396$.

${ }^{4}$ Holt, L. E. Jr. and Snydemann, S. E. Protein and aminoacid requirements of infants and children. Nutr. Albstr. Rer. $35 \%$ $1-13,1965$.

"Bata, L. J,; Kromol, R.; Urrutia, J.; Garcia, B. EfTect of infectim on food intake and the mutrition state. perspectives as viewed fron the village. Am. J. Clin. Nutr. 30: 1215-1227, 1977.

${ }^{6}$ Eurke. J A., Giardiasis in childhood. Am. J. Diseases Children 129: 1304, 1975.

${ }^{7}$ Rusenbert, L.H.; Beiset, W. R.; Gordon, J.E.; Katz, M.; Keush, C. T.; Lucke, T. D., y Mat $t, L$. J. Infast and child enteritismalabsortion-malnutritnun: the potential of limited studies with low-duse: antibiotic feeding. Am. J. Clin. Nutr. 27: 304, 1974.

8 Deacys William, D. Anorexia in cancer Patients. Cancer Research 37: 2354-2358. July 1977. Part 2.

${ }^{9}$ Tabla de: Composición Quimica de Alimentos Chilenos. Facultad de Ciencias Quínicas y Farmacológicas. U. de Chile. 6. Bjodición 1979
${ }^{10}$ Recomeaded Dietary Alowances. National Research CouncilNational acadeny of Sciences. Eight Hevised. Edition 1974.

1 t Feigin Ralph, D. Resume of the discussion concerring recommendations for dietary intake during infection. Am. J. Clin. Nutr. 30: 1548-1551, 1977 .

12 Association of Official Agriculhural Chemists Official methods of Analysis if the AOAC 1 Ith ed. Washingtom, D.C. The Association 1970.

13 Jellife, D. B. Evaluación del estado de autrición de la comunidad, Ginebra, Organización Mundial de la Salud, editores 1974.

14 Rajkin, M. L, Olguin de Marieni, M. C.; Despo, G. A., y Sosa, C.F. "Fraccionamiento proteico por determiruchn directe de albúmina". Biocpuimica Clínica V: II 4: 241, 1974.

15 Araya, H. y Arroyave, G. Pelación del contenido energético praveniente de grasas y protefnas como indicador de patencialidad energética-proteinica de las dietas de poblaciones. Arch. Latimoamer. de Nutr. 29: \{1\} 103-112.

16 Beafon, G. y Stuiss, L. D. Evaluation of the nutritional quality of food supplies: Prediction of desirable or safe Proteix: Calorie ratios. Am. J. Clun. Nutr. 27: 485504, 1974.

${ }^{17}$ Fomon, S. J. InEant Nutrition Ed, 2 Philadelphia W.B. Sanders Co., 1974.

th Uauy, R. y Guzitua, $R$. Evaluación del estado nutricioneal del enfermo hospitalizado. Revista Médica de Chile, l08: 542 , 1980. 